\title{
Inference for the median residual life function in sequential multiple assignment randomized trials
}

\author{
Kelley M. Kidwell, ${ }^{\mathrm{a} \dagger}{ }^{\dagger}$ Jin H. Ko ${ }^{\mathrm{b}}$ and Abdus S. Wahed ${ }^{\mathrm{c}}$
}

\begin{abstract}
In survival analysis, median residual lifetime is often used as a summary measure to assess treatment effectiveness; it is not clear, however, how such a quantity could be estimated for a given dynamic treatment regimen using data from sequential randomized clinical trials. We propose a method to estimate a dynamic treatment regimen-specific median residual life (MERL) function from sequential multiple assignment randomized trials. We present the MERL estimator, which is based on inverse probability weighting, as well as, two variance estimates for the MERL estimator. One variance estimate follows from Lunceford, Davidian and Tsiatis' 2002 survival function-based variance estimate and the other uses the sandwich estimator. The MERL estimator is evaluated, and its two variance estimates are compared through simulation studies, showing that the estimator and both variance estimates produce approximately unbiased results in large samples. To demonstrate our methods, the estimator has been applied to data from a sequentially randomized leukemia clinical trial. Copyright $($ ) 2013 John Wiley \& Sons, Ltd.
\end{abstract}

Keywords: dynamic treatment regimen; adaptive treatment strategy; inverse probability weighting; median residual life function; sequential randomization; non-parametric estimation

\section{Introduction}

Patients are often interested in a summary measure of their prognosis or survival throughout treatment. At diagnosis, physicians may explain the benefit of a particular treatment by giving an estimate of the patient's expected survival he/she receives that treatment. Physicians may choose to present the survival estimates based on Kaplan-Meier curves from large randomized controlled trials by the mean or median time depending on the nature of the data. Patients are likely to request updated survival times as they progress through their treatment(s), such that if they have already survived to some point, they may be interested in their residual life expectancy following a particular treatment plan. These residual estimates are of increasing importance as newer and more personalized treatments become available and as physicians tailor sequences of treatments to individuals over time. As treatment becomes more personalized and sequential such that subsequent treatment may depend on the individual's response to previous treatment, on the course of the individual's disease, or on characteristics or behaviors of the individual, patients and physicians have particular interest in the residual lifetime of the entire treatment regimen, not just the last treatment in an individualized sequence.

Sequences of treatments tailored to the individual are called dynamic treatment regimens (DTRs), also known as adaptive treatment strategies. DTRs are particularly valuable for treating chronic diseases, such as cancer, depression, and AIDS, where treatment is frequently modified to adapt to patients' health status. The concept of DTRs is motivated by the fact that when patients take one treatment after another, especially in the setting of chronic diseases, it is often the entire sequence of treatments which yields the overall outcome, not just the most recent treatment.

${ }^{a}$ Department of Biostatistics, School of Public Health, University of Michigan, Ann Arbor, MI 48109, U.S.A.

${ }^{b}$ Novartis Pharmaceuticals Corporation, East Hanover, NJ, U.S.A.

${ }^{c}$ Department of Biostatistics, Pitt Public Health, University of Pittsburgh, Pittsburgh, PA 15261, U.S.A.

*Correspondence to: Kelley M. Kidwell, University of Michigan, Department of Biostatistics, Ann Arbor, MI, U.S.A.

†E-mail:kidwell@umich.edu 
To elaborate on the concept of DTRs and illustrate the difference between comparing treatments at one stage in the disease process (local comparison) and comparing sequences of treatments throughout the disease process (global comparison), consider the setting of acute myeloid leukemia (AML). DTRs are particularly suitable in the treatment of AML due to two main questions of interest: (i) should induction chemotherapy be followed with a growth factor infusion, and (ii) if a patient achieves remission, should maintenance therapy consist of a mono-therapy or combination therapy. These questions have been addressed locally by comparing survival between treatment groups at specific stages of the disease/trial. We can construct DTRs including the first and second stage treatments, as well as, the intermediate remission status and then ask global questions, such as what is the best treatment regimen to achieve the best overall survival.

To construct a DTR, we put the stages of treatment and any intermediate decisions together. An example of a DTR in the treatment of AML is 'Treat the patient with induction chemotherapy and add a growth factor; if remission is achieved, treat with a combination of maintenance therapies; if remission is not achieved, declare a treatment failure.' Another regimen may be stated in the same way, but eliminating the growth factor. Thus, regimens are not just a sequence of treatments, but they also contain a decision rule based on individual characteristics or response that dictates specific treatments (or lack thereof) in the sequence. Estimation of the entire DTR, including the initial treatment, response, and maintenance therapy, is of interest to find the best global treatment for a patient.

Locally, in the treatment of AML, there are two comparisons, where the first consists of comparing induction treatments of chemotherapy alone versus chemotherapy plus a growth-stimulating factor, and the second consists of comparing maintenance treatments of mono-therapy versus combination therapy. The local comparisons may shine light on the best treatment at that stage for a specific group of people, but combining the conclusions from the two local comparisons may not be the best globally. For example, the best first-stage treatment for achieving remission may not produce the best extended survival (perhaps the first-stage treatment was so strong or produced toxicities so that the maintenance treatment could not be tolerated). Moreover, the best maintenance treatment discovered in isolation may act synergistically or antagonistically with a particular first-stage treatment. Because the treatment would likely be prescribed and followed as a DTR in practice and we are ultimately interested in the overall result, it is important to estimate and compare the effect of DTRs. A global metric for identifying the best DTR for AML or any chronic disease may be the expected residual lifetime at a specific time (e.g., one year).

Sequential multiple assignment randomized trials (SMARTs) [1,2] are designed to follow patients over time, develop, and potentially compare DTRs. In SMARTs, patients are randomized to treatment options and re-randomized to move from one stage to another as the trial progresses. Figure 1 shows an example of a two-stage SMART from the Cancer and Leukemia Group B (CALGB) co-operative group for the treatment of leukemia [3,4]. At entry, patients were randomized to one of the two firststage treatments, standard chemotherapy $\left(A_{1}\right)$ or standard chemotherapy plus granulocyte-macrophage colony-stimulating factor (GM-CSF, $A_{2}$ ). If the patient achieved complete remission, he or she was randomized to one of the second-stage treatments, cytarabine $\left(B_{1}\right)$ or cytarabine plus mitoxantrone $\left(B_{2}\right)$. Because this trial was conducted in a sample of elderly patients, the treatment was considered a failure if remission was not achieved and no further treatment was provided. This SMART contains four embedded DTRs $A_{j} B_{k}, j, k=1,2$, where the DTR $A_{j} B_{k}$ is defined as 'Treat with $A_{j}$ followed by $B_{k}$ if a response to $A_{j}$ is observed, otherwise there is no further treatment' [5]. Note that a patient belonging to either of the following two scenarios is considered to be treated (or consistent) with DTR $A_{j} B_{k}$ :

(1) patient received $A_{j}$ and did not respond and,

(2) patient received $A_{j}$, responded, and received $B_{k}$ at the second-stage.

Throughout such a trial, or more generally, throughout treatment, physicians and patients are interested in an easy to understand summary measure of benefit. For survival analysis, patient benefit is frequently measured by the survival probability or often by the sample mean or median survival time.

The estimation of mean survival time in a SMART, mainly in two-stage designs, has been studied extensively in statistical literature. Lunceford, Davidian and Tsiatis (LDT) [5] proposed estimators for the survival distribution and mean restricted survival time of different treatment regimens using the concept of inverse probability weighting [6]. By including additional information from auxiliary covariates, Wahed and Tsiatis $[7,8]$ improved upon the efficiency of such estimators presenting locally efficient estimators for complete and right-censored cases. Guo and Tsiatis [9] proposed an easier to compute and 


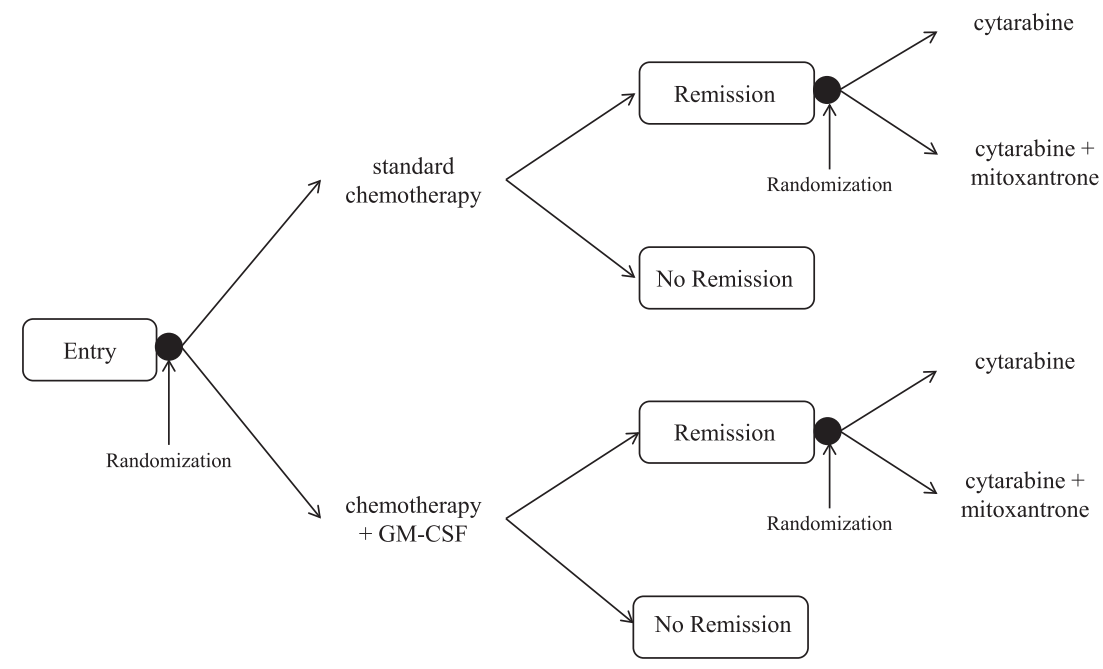

Figure 1. An example of sequential multiple assignment randomized trial from Cancer and Leukemia group B including four embedded dynamic treatment regimens.

efficient weighted risk set estimator to summarize survival distributions of DTRs using time-dependent weights and the Nelson-Aalen estimator.

The aforementioned methods summarized survival distributions from SMARTs using estimated population means, but this measure is not always the best summary. The sample mean is not necessarily representative of the overall survival distribution when data are highly skewed. In such cases, percentiles, including the median, are more useful to outline survival distributions. The median residual life (MERL) function is frequently used as an alternative to the mean summary of time-to-event data. The MERL estimate at a given time point addresses patients' and physicians' request for an updated synopsis of survival, given that the patient has survived up to that point.

The MERL function is the median of the remaining lifetime at a specific time point, and its estimation and comparison between two groups have been well researched in standard one-stage trials. For overall survival $T$, the median residual lifetime at time $t_{0}$ is defined as

$$
\theta\left(t_{0}\right)=\operatorname{median}\left(T-t_{0} \mid T \geqslant t_{0}\right),
$$

where median $(X \mid Y)$ stands for the median of the conditional distribution of $X$ given $Y$. In other words, $\theta\left(t_{0}\right)$ is the median survival time for those who survived beyond time $t_{0}$ in the population. The MERL is stable because it is not affected by outliers (such as extended durations of survival time). In the case of censored data, using the median residual lifetime not only exhibits a better location estimate than using the mean, but it also shows less sensitivity to skewed distributions. In order for the MERL to be defined under censoring at least half of those remaining must have observed failure times [10].

Various methods have been proposed to estimate median residual lifetimes for one and two sample cases for one-stage randomized clinical trials. Haines and Singpurwalla [11] introduced the general concept of the $\alpha$-percentile residual life function $(0<\alpha<1)$. This concept was discussed and expanded upon in Arnold and Brokett [12], Joe and Proschan [13], Joe [14], and Song and Cho [15]. Csorgo and Csorgo [16] proposed a $100(1-p)$ th percentile residual lifetime estimator for complete data, whereas Chung [17] incorporated censoring. A non-parametric estimator of the 100(1-p)th percentile residual lifetime was proposed by Feng and Kulasekera [18] using an inverse function of the Kaplan-Meier curve. Lillo [19] provided a thorough background of statistical literature related to the MERL function, offered a different approach from Joe and Proschan [13] to characterize the family of distributions, and explored relationships between the analytical properties of survival and MERL functions. These methods, however, cannot be applied to estimate regimen-specific median residual lifetimes in two-stage SMART designs because they do not account for the sequential randomization structure.

The MERL function has not been estimated in the setting of SMARTs and development of DTRs. In this paper, we develop a method for the non-parametric estimation of a regimen-specific MERL function from two-stage sequential randomization trials which can be generalized to the estimation of percentiles other than the median. The method utilizes marginal mean models for survival data introduced by LDT 
[5]. Section 2 describes the notation used throughout this article. Section 3 develops the MERL function estimate and two corresponding variance estimate methods. Section 4 presents simulation studies demonstrating the performance of our proposed method and variance estimates. Section 5 applies the method to the CALGB leukemia data set to estimate the MERL function of four DTRs. We conclude with a discussion in Section 6.

\section{Notation and assumptions}

To estimate the MERL function of DTRs, we consider the two-stage design described in the previous section. The development of our method uses the concept of counterfactual variables [20] borrowed from causal inference literature. To introduce this concept and our notation, consider only the population that receives the initial treatment $A_{1}$ (development for the population receiving $A_{2}$ is analogous). For the $i^{\text {th }}$ individual in the population, let $R_{i}$ be the observed response indicator: $R_{i}=1$ if patient $i$ responds to the first-stage treatment $A_{1}, R_{i}=0$ otherwise. If patient $i$ did not respond, his or her survival time is denoted by $T_{N R i}$. If patient $i$ responded and received maintenance treatment $B_{k}$, his or her time to the start of the second-stage treatment is denoted by $T_{R i}$ and his or her post-response (or post-start-ofsecond-stage) survival time is denoted by $T_{k i}^{*}$. Therefore, under the DTR $A_{1} B_{k}$, the overall survival time for patient $i$ can be defined as

$$
T_{k i}=\left(1-R_{i}\right) T_{N R i}+R_{i}\left(T_{R i}+T_{k i}^{*}\right), k=1,2 .
$$

Note that, $T_{N R i}, T_{1 i}$, and $T_{2 i}$ cannot all be observed for the same patient because patient $i$ either did not respond to the initial treatment and therefore did not receive any maintenance therapy $\left(T_{k i}=T_{N R i}\right.$, $k=1,2$ ), or patient $i$ responded and received only one of the two maintenance therapies, $B_{1}$ $\left(T_{1 i}=T_{R i}+T_{1 i}^{*}\right)$ or $B_{2}\left(T_{2 i}=T_{R i}+T_{2 i}^{*}\right)$. These variables, $T_{1 i}$ and $T_{2 i}$, are referred to as counterfactuals or potential outcomes [20]. Counterfactuals include the set of all possible outcomes, in this case survival times, including those which we observe and those which we do not.

Our interest lies in estimating the MERL function for DTR $A_{1} B_{k}, k=1,2$, or equivalently, the MERL function for the overall survival $T_{k i}$. Now, if the survival time for the $i^{t h}$ patient, $T_{i}$, is observed, it can be expressed in terms of $T_{1 i}$ and $T_{2 i}$ by means of the consistency assumption [21], such that, $T_{i}=Z_{1 i} T_{1 i}+Z_{2 i} T_{2 i}$, where $Z_{k i}, k=1,2$, is the $B_{k}$ treatment assignment indicator, i.e., $Z_{k i}=1$ if the $i^{\text {th }}$ patient was assigned to treatment $B_{k}, 0$ otherwise. Alternatively, $T_{i}$ can be expressed as,

$$
T_{i}=\left(1-R_{i}\right) T_{N R i}+R_{i}\left(T_{R i}+Z_{1 i} T_{1 i}^{*}+Z_{2 i} T_{2 i}^{*}\right) .
$$

However, because of censoring, $T_{i}$ may not necessarily be observed. The observed data from a two-stage design similar to that described in Figure 1 can be denoted by $\left(R_{i}, R_{i} Z_{k i}, U_{i}, \Delta_{i}\right), i=1 \ldots n ; k=1,2$, where $R_{i}$ and $Z_{k i}$ are defined previously, $\Delta_{i}$ is the complete case indicator, and $U_{i}$ is the event (survival or censoring) time. If $C_{i}$ is used to denote the censoring time for the $i^{\text {th }}$ individual in the sample, then $U_{i}=\min \left(T_{i}, C_{i}\right)$ and $\Delta_{i}=I\left(T_{i}<C_{i}\right)$. Note that, in practice, patients may be censored prior to observing a response; such patients are historically treated as non-responders in the analysis. We assume that censoring does not depend on $T_{i}$ or any other observed or counterfactual data.

Let $K(t)$ be the survival distribution function of censoring time $C_{i}$, i.e., $K(t)=P\left(C_{i} \geqslant t\right)$ and let $\pi_{1}=P\left(Z_{1 i}=1 \mid R_{i}=1\right)$ and $\pi_{2}=1-\pi_{1}$ denote the probability of receiving treatment $B_{1}$ or $B_{2}$, respectively. Further, let $S_{k}(t)=P\left(T_{k}>t\right)$ denote the survival probability at time $t$ under regimen $A_{1} B_{k}$ for $k=1,2$. Then, our goal is to find an estimator of the MERL under DTR $A_{1} B_{k}$ for $k=1,2$

$$
\theta_{k 0}=\theta_{k}\left(t_{0}\right)=\operatorname{median}\left(T_{k}-t_{0} \mid T_{k}>t_{0}\right) .
$$

\section{Estimation of the median residual life function}

\subsection{Median residual life estimator}

We first express $\theta_{k 0}$ as a function of $S_{k}\left(t_{0}\right)$ so that the median residual lifetime can be indirectly estimated from the estimated survival curve. The MERL defined in Equation (1) can be equivalently expressed as $P\left(T_{k}-t_{0} \geqslant \theta_{k 0} \mid T_{k} \geqslant t_{0}\right)=\frac{1}{2}$, thereby suggesting that $\theta_{k 0}=S_{k}^{-1}\left[\frac{1}{2} S_{k}\left(t_{0}\right)\right]-t_{0}$, where $S_{k}^{-1}(u)$ is the inverse of the survival function at $u$, i.e., $S_{k}^{-1}(u)=\inf \left[t: S_{k}(t)<u\right]$. 
Thus, if we consistently estimate the survival distribution $S_{k}\left(t_{0}\right)$ by $\hat{S}_{k}(t)[18,22]$, we can estimate $\theta_{k 0}$ by

$$
\hat{\theta}_{k 0}=\hat{S}_{k}^{-1}\left[\frac{1}{2} \hat{S}_{k}\left(t_{0}\right)\right]-t_{0}
$$

This method of estimation is valid as long as $\hat{S}_{k}^{-1}(t)$ exists at $t=\frac{1}{2} \hat{S}_{k}\left(t_{0}\right)$.

To consistently estimate the survival curve for a given DTR $A_{1} B_{k}, k=1,2$, we use the weight-normalized inverse probability-weighted estimator from Lunceford, Davidian, and Tsiatis [5] defined as:

$$
\hat{S}_{k}\left(t_{0}\right)=\left[\sum_{i=1}^{n} \frac{\Delta_{i} Q_{k i}}{\hat{K}\left(U_{i}\right)}\right]^{-1}\left[\sum_{i=1}^{n} \frac{\Delta_{i} Q_{k i}}{\hat{K}\left(U_{i}\right)} I\left(U_{i}>t_{0}\right)\right],
$$

where $\hat{K}\left(U_{i}\right)$ is the Kaplan-Meier estimator of $K(t)$ and $Q_{k i}=1-R_{i}+\frac{R_{i} Z_{k i}}{\pi_{k}}$ is the weight function. In estimating the survival function $S_{k}$, every uncensored survival time $U_{i}$ is weighted by $\frac{1}{K\left(U_{i}\right)}$ to reflect the fact that there were $\frac{P\left(C_{i} \leq U_{i}\right)}{P\left(C_{i}>U_{i}\right)}=\frac{1}{K\left(U_{i}\right)}-1$ expected censored individuals in the population at time $U_{i}$. Therefore, an uncensored individual counts for himself or herself and $\frac{1}{K\left(U_{i}\right)}-1$ additional censored individuals. The weight function, $Q_{k i}$, assigns each observation the appropriate weight consistent with DTR $A_{1} B_{k}, k=1,2$. More specifically, $Q_{k i}$ is defined to reflect that patients who do not respond to $A_{1}$ are consistent with both DTRs $A_{1} B_{1}$ and $A_{1} B_{2}$, and only a portion of patients who respond are treated with $A_{1} B_{1}\left(A_{1} B_{2}\right)$ with randomization probability $\pi_{1}\left(\pi_{2}\right)$. Thus, for the treatment regimen $A_{1} B_{1}, Q_{1 i}=0$ if individual $i$ responded to $A_{1}$ and received $B_{2}$ (i.e., $R_{i}=1$ and $Z_{i}=0$ ), $Q_{1 i}=1$ if individual $i$ did not respond to $A_{1}\left(R_{i}=0\right)$, and $Q_{1 i}=\pi_{1}^{-1}$ if individual $i$ responded to $A_{1}$ and received $B_{1}\left(R_{i}=1\right.$ and $Z_{i}=1$ ). Similarly, $Q_{2 i}$ acts as the weight when an individual receives treatment consistent with DTR $A_{1} B_{2}$.

By using this consistent estimate of the survival function for each DTR, we obtain the estimator of the MERL function, $\hat{\theta}_{k 0}$, by substituting Equation (3) in Equation (2). In the remaining subsections, we propose two different methods for estimating the variance of $\theta_{k 0}$.

\subsection{Lunceford, Davidian and Tsiatis variance estimator}

The first variance estimator follows directly from the survival function-based variance estimator from LDT [5]. We mention again that $\hat{\theta}_{k 0}$ satisfies

$$
\hat{S}_{k}\left[t_{0}+\hat{\theta}_{k 0}\right]-\frac{1}{2} \hat{S}_{k}\left(t_{0}\right)=0
$$

Let us denote the left hand side of Equation (4) by $\hat{M}_{k}\left(\hat{\theta}_{k 0}\right)$. This quantity, $\hat{M}_{k}\left(\hat{\theta}_{k 0}\right)$, is a random variable even if $\theta_{k 0}$ is known due to the estimated survival function $\hat{S}_{k}(t)$. Expanding $\hat{M}_{k}\left(\hat{\theta}_{k 0}\right)$ around $\theta_{k 0}$, we obtain,

$$
\hat{M}_{k}\left(\hat{\theta}_{k 0}\right) \approx \hat{M}_{k}\left(\theta_{k 0}\right)+\left(\hat{\theta}_{k 0}-\theta_{k 0}\right) \frac{\partial \hat{M}_{k}\left(\theta_{k 0}\right)}{\partial \theta_{k 0}} .
$$

Assuming sufficient regularity, it follows that variance of $\hat{\theta}_{k 0}$ can be approximated by

$$
\operatorname{var}\left(\hat{\theta}_{k 0}\right) \approx \frac{\operatorname{var}\left[\hat{M}_{k}\left(\theta_{k 0}\right)\right]}{\mathrm{E}\left[\frac{\partial \hat{M}_{k}\left(\theta_{k 0}\right)}{\partial \theta_{k 0}}\right]^{2}}
$$

Note that $\frac{\partial \hat{M}_{k}(\theta)}{\partial \theta}=-\hat{f}_{k}\left(t_{0}+\theta\right)$, leading to

$$
\operatorname{var}\left(\hat{\theta}_{k 0}\right) \approx \frac{\operatorname{var}\left[\hat{M}_{k}\left(\theta_{k 0}\right)\right]}{\hat{f}_{k}^{2}\left(t_{0}+\theta_{k 0}\right)},
$$

where $f_{k}(\cdot)$ is the density function of $T_{k}$, which can be estimated by using an inverse probabilityweighted non-parametric smoothing technique such as the kernel density estimator. 
To estimate the variance in the numerator in Equation (5), note that $\hat{M}_{k}\left(\theta_{k 0}\right)$ can be written as

$$
\left[\sum_{i=1}^{n} \frac{\Delta_{i} Q_{k i}}{\hat{K}\left(U_{i}\right)}\right]^{-1}\left[\sum_{i=1}^{n} \frac{\Delta_{i} Q_{k i}}{\hat{K}\left(U_{i}\right)} h\left(U_{i} ; \theta_{k 0}\right)\right]
$$

where $h\left(U_{i} ; \theta\right)=I\left(U_{i}>t_{0}+\theta\right)-\frac{1}{2} I\left(U_{i}>t_{0}\right)$. This is very similar to the survival function estimate in LDT [5] presented in Equation (3) except that $I\left(U_{i}>t_{0}\right)$ is replaced by $h\left(U_{i} ; \theta_{k 0}\right)$. They show that the estimator $\hat{S}_{k}\left(t_{0}\right)$ defined by Equation (3) is a consistent and asymptotically normal estimator with mean $S_{k}\left(t_{0}\right)$ and variance $\sigma_{k}^{2}\left(t_{0}\right)$, that can be estimated by

$$
\hat{\sigma}_{k}^{2}\left(t_{0}\right)=\frac{1}{n}\left\{\frac{1}{n} \sum_{i=1}^{n} \frac{\Delta_{i}}{\hat{K}\left(U_{i}\right)}\left[Q_{k i} I\left(U_{i}>t_{0}\right)-\hat{S}_{k}\left(t_{0}\right)\right]^{2}+\int_{0}^{L} \frac{d N^{c}(u)}{\hat{K}(u) Y(u)} \hat{\mathrm{E}}\left[L_{k i}(t, u)\right]^{2}\right\},
$$

where,

$$
\hat{\mathrm{E}}\left[L_{k i}(t, u)\right]^{2}=\frac{1}{n} \sum_{i=1}^{n} \Delta_{i}\left\{Q_{k i}\left[I\left(U_{i}>t_{0}\right)-\hat{S}_{k}\left(t_{0}\right)\right]-\hat{G}_{k}(t, u)\right\}^{2} \frac{I\left(U_{i} \geqslant u\right)}{\hat{K}\left(U_{i}\right)}
$$

and

$$
\hat{G}_{k}(t, u)=\frac{1}{n \hat{S}(u)} \sum_{i=1}^{n} \Delta_{i} Q_{k i}\left[I\left(U_{i}>t_{0}\right)-\hat{S}_{k}\left(t_{0}\right)\right] \frac{I\left(U_{i} \geqslant u\right)}{\hat{K}\left(U_{i}\right)} .
$$

Here, $N^{c}(u)=\sum_{i=1}^{n} N_{i}^{c}(u)=\sum_{i=1}^{n} I\left(U_{i} \leqslant u, \Delta_{i}=0\right)$ and $Y(u)=\sum_{i=1}^{n} Y_{i}(u)=\sum_{i=1}^{n} I\left(U_{i} \geqslant u\right)$.

A similar derivation can be used to obtain the variance estimator of the variance of $\hat{M}_{k}\left(\theta_{k 0}\right)$, namely,

$$
\begin{aligned}
\operatorname{var}\left[\hat{M}_{k}\left(\theta_{k 0}\right)\right]=\frac{1}{n} & \left\{\frac{1}{n} \sum_{i=1}^{n} \frac{\Delta_{i}}{\hat{K}\left(U_{i}\right)}\left[Q_{k i} h\left(U_{i} ; \hat{\theta}_{k 0}\right)-\hat{M}_{k}\left(\hat{\theta}_{k 0}\right)\right]^{2}\right. \\
& \left.+\int_{0}^{L} \frac{d N^{c}(u)}{\hat{K}(u) Y(u)} \hat{\mathrm{E}}\left[L_{k i}(t, u)\right]^{2}\right\}
\end{aligned}
$$

where

$$
\hat{\mathrm{E}}\left[L_{k i}(t, u)\right]^{2}=\frac{1}{n} \sum_{i=1}^{n} \Delta_{i}\left[Q_{k i}\left\{h\left(U_{i} ; \hat{\theta}_{k 0}\right)-\hat{M}_{k}\left(\hat{\theta}_{k 0}\right)\right\}-\hat{G}_{k}(t, u)\right]^{2} \frac{I\left(U_{i} \geqslant u\right)}{\hat{K}\left(U_{i}\right)}
$$

and

$$
\hat{G}_{k}(t, u)=\frac{1}{n \hat{S}(u)} \sum_{i=1}^{n} \Delta_{i} Q_{k i}\left\{h\left(U_{i} ; \hat{\theta}_{k 0}\right)-\hat{M}_{k}\left(\hat{\theta}_{k 0}\right)\right\} \frac{I\left(U_{i} \geqslant u\right)}{\hat{K}\left(U_{i}\right)} .
$$

Equation (7), along with an inverse probability-weighted kernel density estimator of $f_{k}$, can then be used in Equation (5) to produce a variance estimator of $\hat{\theta}_{k 0}$, which we refer to as the LDT variance estimator.

\subsection{Sandwich variance estimator}

To derive the second variance estimator of $\theta_{k 0}$, note that the MERL estimator given in Equation (2) using the consistent estimate of survival provided in Equation (3) is equivalent to directly solving an appropriate estimating equation of the form

$$
\sum_{i=1}^{n} g_{i}\left(\theta_{k 0}\right)=0
$$

where $g_{i}\left(\theta_{k 0}\right)=\frac{\Delta_{i} Q_{k i}}{K\left(U_{i}\right)}\left[I\left(U_{i}>t_{0}+\theta_{k 0}\right)-\frac{1}{2} I\left(U_{i}>t_{0}\right)\right]$, and $K(\cdot)$ is unknown and must be consistently estimated (we use the Kaplan Meier estimator $\hat{K}\left(U_{i}\right)$ ). 
On the basis of this representation of $\hat{\theta}_{k 0}$, an alternative method to estimating the variance of $\theta_{k 0}$ is to use the sandwich estimator (Stefanski and Boos [23]). Because $g_{i}\left(\theta_{k 0}\right)$ is not a smooth function, the sandwich variance is given by, $\operatorname{var}\left(\theta_{k 0}\right)=n^{-1} A\left(\theta_{k 0}\right)^{-1} B\left(\theta_{k 0}\right)\left[A\left(\theta_{k 0}\right)^{-1}\right]^{T}$, where $A\left(\theta_{k 0}\right)=$ $-\frac{\partial}{\partial \theta_{k 0}} \mathrm{E}\left[g\left(\theta_{k 0}\right)\right]$ and $B\left(\theta_{k 0}\right)=\mathrm{E}\left[g\left(\theta_{k 0}\right) g\left(\theta_{k 0}\right)^{T}\right]$. To estimate these quantities and, hence, the variance of $\hat{\theta}_{k 0}$, we replace $A(\cdot)$ and $B(\cdot)$ by their empirical counterparts and obtain the sandwich variance estimator

$$
\operatorname{var}\left(\hat{\theta}_{k 0}\right)=\frac{B_{n}\left(\hat{\theta}_{k 0}\right)}{n\left[A_{n}\left(\hat{\theta}_{k 0}\right)\right]^{2}}
$$

where $A_{n}\left(\hat{\theta}_{k 0}\right)=-\hat{f}_{k}\left(t_{0}+\hat{\theta}_{k 0}\right)$ and $B_{n}\left(\hat{\theta}_{k 0}\right)=\left[\sum_{i=1}^{n} \frac{\Delta_{i} Q_{k i}}{\hat{K}\left(U_{i}\right)}\right]^{-1} \sum_{i=1}^{n}\left[g_{i}\left(\hat{\theta}_{k 0}\right)\right]^{2}$.

\section{Simulation study}

We conducted simulation studies to evaluate the proposed MERL estimator and to compare the LDT and sandwich variance estimators. The simulations were designed for the induction treatment $A_{1}$. Because initial treatment $A_{1}$ or $A_{2}$ is assigned by randomization, results for DTRs beginning with $A_{2}$ are analogous. We generated $n$ independent sets of observations as follows. An indicator of the patient's response, $R_{i}$, was generated from a Bernoulli $\left(\pi_{R}\right)$ distribution, where $\pi_{R}$ was set to be either 0.4 or 0.7. For responders $\left(R_{i}=1\right)$, the $B_{1}$ treatment assignment indicator, $Z_{1 i}$, was generated from a Bernoulli distribution with $\pi_{1}=0.3$ or $\pi_{1}=0.5$. We then defined $Z_{2 i}=1-Z_{1 i}$. For non-responders $\left(R_{i}=0\right)$, we generated survival time $T_{N R i}$ from an exponential distribution with mean $\lambda=1$, truncated at $b=3$. For responders, a response time, $T_{R i}$, was generated from an exponential distribution with mean $\alpha=1$, truncated at $b_{\alpha}=3.5$. For patients receiving maintenance treatment $B_{k}$, post-remission survival time $T_{k i}^{*}$ was generated from the relationship $T_{k i}^{*}=T_{R i}+\left(\beta_{1}+\beta_{2} T_{R i}\right) u_{k i}$, for $k=1,2$, where $\beta_{1}=\beta_{2}=1$ and $u_{k i}$ are generated from a uniform distribution from 0 to $\gamma_{k}$, where $\gamma_{1}=1$ and $\gamma_{2}=1$.5. Finally, the survival time for the $i^{t h}$ patient is defined as $T_{i}=\left(1-R_{i}\right) T_{N R i}+R_{i}\left(T_{R i}+Z_{1 i} T_{1 i}^{*}+Z_{2 i} T_{2 i}^{*}\right)$. Various censoring times, $C_{i}$, were taken from a uniform distribution between 0 and $V$ to reflect about $4-40 \%$ censoring. For the specific simulations in Tables I and II, $V=7.4$ for censoring of $17 \%$ when $\pi_{R}=0.4$ and $21 \%$ when $\pi_{R}=0.7$. Censoring was assumed independent of all other variables. The observed survival is defined as $U_{i}=\min \left(T_{i}, C_{i}\right)$, and the complete-case indicator is $\Delta_{i}=I\left(T_{i}<C_{i}\right)$.

We generated 5000 samples of size $n$ (250 and 500) under different scenarios to estimate the median residual lifetime of DTRs $A_{1} B_{1}$ and $A_{1} B_{2}$ at 183.625 and 365.25 days (one half of a year and 1 year). We summarize the results by presenting the estimate of the MERL with the standard error (SE) estimates using the LDT and sandwich methods, and the corresponding 95\% coverage probabilities (CPs) in Tables I and II.

Table I presents results from the scenarios where patients were assigned to the maintenance treatments, $B_{1}$ and $B_{2}$, with equal allocation $\left(\pi_{1}=\pi_{2}=0.5\right)$. We see that the estimator is approximately unbiased with small observed biases that decrease with increasing sample sizes. The bias is at most $0.7 \%$ for $n=500$. Sandwich SEs are slightly larger than the LDT SEs. In most cases, the MERL estimator with either variance achieved the nominal level of CP $(95 \%)$.

Scenarios with unequal probability of assignment to maintenance treatments, $B_{1}$ and $B_{2}$, are shown in Table II. Here $\pi_{1}=0.3$, so $30 \%$ of the responders were assigned to $B_{1}$, while $70 \%$ were assigned to $B_{2}$. Performance of the estimator, CPs, and SEs remained similar to results in Table I with equal randomization. With unequal randomization, however, MERL estimators for DTRs corresponding to the maintenance treatment with higher probability of randomization have smaller SEs compared with that for DTRs containing maintenance treatment with lower probability of randomization. Specifically, SEs (LDT or sandwich) for $A_{1} B_{1}$ were larger than that for $A_{1} B_{2}$ and larger than the SEs in Table I with 50\% randomization, when $30 \%$ of responders were assigned to $B_{1}$. This is expected because this form of randomization increases the effective sample size of regimen $A_{1} B_{2}$ and reduces the number of patients following DTR $A_{1} B_{1}$.

As censoring increases, the bias generally increases, as do the SEs for smaller sample sizes (simulations not shown assessed censoring up to 40\%). As censoring increases, the effective sample size decreases as we have less patients with full information, so a similar pattern is seen between increasing censoring and decreasing sample size. However, particularly for sample size of 500, the MERL and its 


\begin{tabular}{|c|c|c|c|c|c|c|c|c|c|c|}
\hline \multirow[b]{2}{*}{$\mathrm{n}$} & \multirow[b]{2}{*}{$\pi_{R}$} & \multirow[b]{2}{*}{$t_{0}$} & \multirow[b]{2}{*}{ Strategy } & \multirow[b]{2}{*}{$\theta\left(t_{0}\right)$} & \multirow[b]{2}{*}{ MERL } & \multirow[b]{2}{*}{ MCSE } & \multicolumn{2}{|c|}{ LDT } & \multicolumn{2}{|c|}{ Sandwich } \\
\hline & & & & & & & SE & $\mathrm{CP}$ & SE & $\mathrm{CP}$ \\
\hline \multirow[t]{8}{*}{250} & 0.4 & 183.625 & $A_{1} B_{1}$ & 361 & 362.4 & 48.99 & 47.98 & 0.943 & 50.57 & 0.946 \\
\hline & & & $A_{1} B_{2}$ & 304 & 305.4 & 42.01 & 40.81 & 0.945 & 42.32 & 0.946 \\
\hline & & 365.25 & $A_{1} B_{1}$ & 332 & 335.4 & 59.79 & 57.56 & 0.941 & 60.44 & 0.935 \\
\hline & & & $A_{1} B_{2}$ & 282 & 283.9 & 53.68 & 50.70 & 0.939 & 52.42 & 0.928 \\
\hline & 0.7 & 183.625 & $A_{1} B_{1}$ & 459 & 463.4 & 59.73 & 59.13 & 0.951 & 62.18 & 0.951 \\
\hline & & & $A_{1} B_{2}$ & 360 & 364.8 & 51.55 & 49.31 & 0.939 & 50.96 & 0.943 \\
\hline & & 365.25 & $A_{1} B_{1}$ & 406 & 411.5 & 70.41 & 66.88 & 0.938 & 70.06 & 0.929 \\
\hline & & & $A_{1} B_{2}$ & 328 & 332.7 & 60.61 & 58.46 & 0.943 & 60.26 & 0.931 \\
\hline \multirow[t]{8}{*}{500} & 0.4 & 183.625 & $A_{1} B_{1}$ & 361 & 362.0 & 34.00 & 33.94 & 0.948 & 35.72 & 0.955 \\
\hline & & & $A_{1} B_{2}$ & 304 & 305.3 & 29.75 & 29.06 & 0.941 & 30.08 & 0.944 \\
\hline & & 365.25 & $A_{1} B_{1}$ & 332 & 333.4 & 41.75 & 40.66 & 0.945 & 42.62 & 0.949 \\
\hline & & & $A_{1} B_{2}$ & 282 & 283.4 & 37.29 & 36.05 & 0.943 & 37.19 & 0.938 \\
\hline & 0.7 & 183.625 & $A_{1} B_{1}$ & 459 & 460.3 & 41.86 & 41.55 & 0.947 & 43.65 & 0.953 \\
\hline & & & $A_{1} B_{2}$ & 360 & 361.5 & 35.70 & 34.92 & 0.945 & 36.06 & 0.946 \\
\hline & & 365.25 & $A_{1} B_{1}$ & 406 & 407.6 & 49.38 & 47.62 & 0.941 & 49.82 & 0.945 \\
\hline & & & $A_{1} B_{2}$ & 328 & 331.1 & 44.02 & 41.87 & 0.938 & 43.13 & 0.941 \\
\hline
\end{tabular}

LDT, Lunceford, Davidian and Tsiatis; MERL, median residual life; MCSE, Monte Carlo standard error; SE, standard error; $\mathrm{CP}$, coverage probability.

\begin{tabular}{|c|c|c|c|c|c|c|c|c|c|c|}
\hline \multirow[b]{2}{*}{$\mathrm{n}$} & \multirow[b]{2}{*}{$\pi_{R}$} & \multirow[b]{2}{*}{$t_{0}$} & \multirow[b]{2}{*}{ Strategy } & \multirow[b]{2}{*}{$\theta\left(t_{0}\right)$} & \multirow[b]{2}{*}{ MERL } & \multirow[b]{2}{*}{ MCSE } & \multicolumn{2}{|c|}{ LDT } & \multicolumn{2}{|c|}{ Sandwich } \\
\hline & & & & & & & SE & $\mathrm{CP}$ & SE & $\mathrm{CP}$ \\
\hline \multirow{8}{*}{250} & 0.4 & 183.625 & $A_{1} B_{1}$ & 361 & 364.7 & 61.12 & 59.67 & 0.945 & 62.02 & 0.939 \\
\hline & & & $A_{1} B_{2}$ & 304 & 305.1 & 37.73 & 36.17 & 0.940 & 37.91 & 0.949 \\
\hline & & 365.25 & $A_{1} B_{1}$ & 332 & 340.8 & 76.86 & 71.92 & 0.935 & 74.49 & 0.933 \\
\hline & & & $A_{1} B_{2}$ & 282 & 284.3 & 46.51 & 44.46 & 0.935 & 46.46 & 0.931 \\
\hline & 0.7 & 183.625 & $A_{1} B_{1}$ & 459 & 464.8 & 78.04 & 75.57 & 0.944 & 78.20 & 0.937 \\
\hline & & & $A_{1} B_{2}$ & 360 & 361.3 & 42.87 & 41.86 & 0.944 & 43.90 & 0.951 \\
\hline & & 365.25 & $A_{1} B_{1}$ & 406 & 413.6 & 91.86 & 84.93 & 0.937 & 87.66 & 0.915 \\
\hline & & & $A_{1} B_{2}$ & 328 & 331.4 & 52.56 & 49.87 & 0.938 & 52.13 & 0.937 \\
\hline \multirow[t]{8}{*}{500} & 0.4 & 183.625 & $A_{1} B_{1}$ & 361 & 362.3 & 41.00 & 41.80 & 0.955 & 43.33 & 0.949 \\
\hline & & & $A_{1} B_{2}$ & 304 & 304.6 & 26.49 & 25.63 & 0.943 & 26.84 & 0.947 \\
\hline & & 365.25 & $A_{1} B_{1}$ & 332 & 334.1 & 52.92 & 51.10 & 0.943 & 52.78 & 0.934 \\
\hline & & & $A_{1} B_{2}$ & 282 & 282.3 & 32.91 & 31.65 & 0.943 & 33.04 & 0.946 \\
\hline & 0.7 & 183.625 & $A_{1} B_{1}$ & 459 & 462.9 & 54.44 & 53.46 & 0.946 & 55.24 & 0.948 \\
\hline & & & $A_{1} B_{2}$ & 360 & 363.1 & 30.40 & 29.74 & 0.940 & 31.16 & 0.952 \\
\hline & & 365.25 & $A_{1} B_{1}$ & 406 & 409.9 & 63.50 & 61.37 & 0.941 & 63.25 & 0.937 \\
\hline & & & $A_{1} B_{2}$ & 328 & 329.4 & 36.90 & 35.32 & 0.936 & 36.88 & 0.944 \\
\hline
\end{tabular}

LDT, Lunceford, Davidian and Tsiatis; MERL, median residual life; MCSE, Monte Carlo standard error; SE, standard error; $\mathrm{CP}$, coverage probability.

variance estimates were similar to those shown in Tables I and II, with CP hovering around 95\%. Thus, increased censoring adds slightly more bias to the MERL estimate, but increasing sample sizes to 500 or more for a trial with two DTRs produces approximately unbiased estimates with adequate coverage.

In both Tables I and II, both the LDT and sandwich variance estimates became closer and closer to the Monte Carlo variance with increasing sample size implying consistency of both variance estimates. Overall, the LDT variance estimate was smaller than the sandwich variance estimate, but both provided similar CP. 


\section{Analysis of leukemia Cancer and Leukemia Group B 8923 trial}

We applied the proposed methods described in previous sections to estimate the MERL of patients treated with various treatment regimens from a two-stage randomized clinical trial, shown in Figure 1, conducted by the CALGB $[3,4]$. This double-blinded placebo-controlled trial was designed to examine the effects of giving infusions of granulocyte-macrophage colony-stimulating factor (GM-CSF) following induction chemotherapy in AML patients. Patients were initially randomized to GM-CSF $(n=193)$ or placebo $(n=195)$ following treatment with standard chemotherapy. Patients who achieved remission and consented to second-stage treatment (79 out of 193 in GM-CSF and 90 out of 195 in the placebo group) were re-randomized into one of the intensification therapies: cytarabine (denoted by I) and cytarabine plus mitoxantrone (denoted by II). In this process, 37 patients were assigned to cytarabine (I) in the GM-CSF group, whereas 45 patients were assigned to the same maintenance therapy in the placebo group. The remaining patients in each group received cytarabine plus mitoxantrone (II). Therefore, there are four possible DTRs in this trial, namely GM-CSF/I, GM-CSF/II, Placebo/I, and Placebo/II, where for example, 'GM-CSF/I' stands for, 'Add GM-CSF by infusion after chemotherapy and if the patient achieves remission, follow with maintenance therapy cytarabine, otherwise there is no further treatment.'

In Table III, we present the MERL estimator (Equation (2)) at specific time points (150, 250, and 350 days) for the DTRs GM-CSF/I, GM-CSF/II, Placebo/I, and Placebo/II and the LDT and sandwich variance estimates from (Equation (5) and (9)). Similar to the simulation results, the SEs using the sandwich estimator were slightly larger than those of the LDT estimates. The estimated MERL was smallest for the GM-CSF/II regimen, whereas, it was the largest for regimen Placebo/II at all three time points. As the time of interest $\left(t_{0}\right)$ increased, median residual lifetimes decreased, except for the DTR Placebo/II which specified, 'Do not give GM-CSF after chemotherapy, and when remission is achieved treat with the combination maintenance therapy of cytarabine plus mitoxantrone, otherwise there is no further treatment' We note that only $7.0 \%$ of the individuals in this trial were censored, adding to the reliability of the estimates and SEs.

Estimates of the median residual lifetimes over time are presented in Figure 2 for all four DTRs. This figure shows the evolution of the MERL over the duration of the trial. For all regimens, the MERL estimates initially increased, most likely due to the effect of initial treatment. After about 50 days, the MERL estimates began to decrease for the regimens GM-CSF/I, GM-CSF/II, and Placebo/I. Placebo/II shows increasing median residual lifetimes throughout much of the trial which is due to the fact that a number of the patients following this DTR survived for a very long period. This result is consistent with findings from previous studies based on the mean survival or survival curve $([5,7])$, where it has been

\begin{tabular}{|lcccc|}
\hline \multirow{2}{*}{$\begin{array}{l}\text { Table III. Estimated median residual lifetime for Cancer and Leukemia } \\
\text { Group B 8923 data at different days. }\end{array}$} & & \multicolumn{2}{c|}{ Variance } \\
\cline { 3 - 5 } Strategy & $t_{0}$ (days) & MERL & LDT & Sandwich \\
\hline GM-CSF/I & 150 & 312 & 43.8 & 45.1 \\
& 250 & 265 & 46.1 & 47.4 \\
& 350 & 235 & 51.4 & 52.9 \\
\hline GM-CSF/II & 150 & 285 & 41.3 & 42.4 \\
& 250 & 238 & 44.8 & 46.0 \\
& 350 & 182 & 47.5 & 48.9 \\
\hline Placebo/I & 150 & 318 & 44.6 & 46.8 \\
& 250 & 303 & 50.6 & 53.1 \\
& 350 & 272 & 56.4 & 59.2 \\
\hline Placebo/II & 150 & 403 & 79.1 & 102.9 \\
& 250 & 394 & 95.8 & 127.9 \\
& 350 & 499 & 161.0 & 221.3 \\
\hline
\end{tabular}

MERL, median residual life; LDT, Lunceford, Davidian and Tsiatis; GM-CSF, granulocyte-macrophage colony-stimulating factor. 


\section{Statistics}

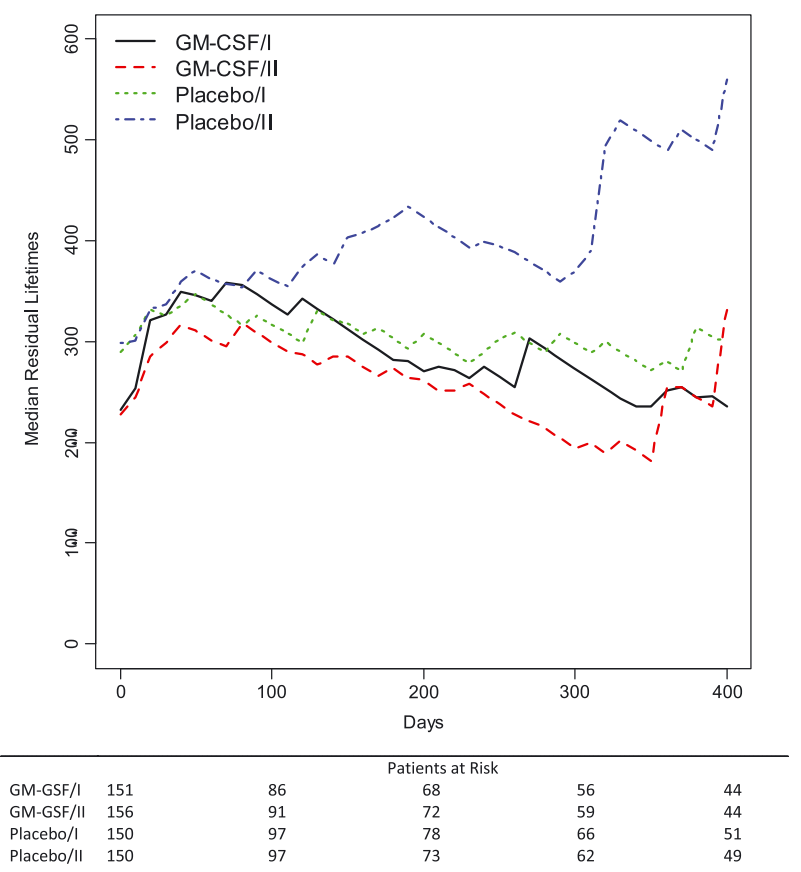

Figure 2. Median residual lifetime estimates for CALGB 8923 data with number of the patients at risk for each dynamic treatment regimen.

shown that infusion with GM-CSF following initial chemotherapy is not beneficial to patients in terms of overall survival.

\section{Discussion}

We presented a non-parametric MERL estimator for sequential multiple assignment randomized trials with two variance estimates. We estimated the MERL indirectly from the estimated survival curve using inverse probability weighting. The first variance estimate, denoted by LDT, was based on the survival function variance estimator from Lunceford, Davidian, and Tsiatis [5]. The second variance estimate was based on the sandwich estimator using the inverse probability estimating equation. The variance estimators differed somewhat with the LDT estimator being slightly more efficient. An important limitation to these variance estimates is that they require the estimation of the density function in order to estimate the variance. When the density estimate is not stable, the variance estimates could be less reliable, and bootstrap samples may be used to estimate the variance. The variance estimates presented, however, are much easier to implement and generally provide approximately unbiased results.

Results from our simulation study show that the MERL estimator is approximately unbiased. The LDT variance estimate has slightly smaller SEs than the sandwich estimate, but the MERL with either variance estimate reaches nominal coverage. It may be of interest to investigate if one particular DTR is statistically significantly better than the others. To address this, one might simply do a Wald-type test of linear contrasts of the treatment strategies. Note that the variance of the estimated contrasts must take into account the dependence of shared-path strategies [24], i.e., regimens that share a common treatment path. Analytical formulas for covariance between shared-path strategies are not as straightforward and are currently being studied. A bootstrap approach could be used to estimate the SEs. Future research in this area might consider including covariates in the estimation process and developing test statistics to compare median residual lifetimes across different DTRs taking into account the possible shared-path nature of the regimens.

\section{Acknowledgements}

This work has stemmed from the PhD dissertation [25] of the second author, Dr. Ko. We thank the dissertation committee members Drs. Jong-Hyeon Jeong, Stewart Anderson, and Yu Cheng for their input. Dr. Wahed's research was in part supported by a National Institute of Mental Health Grant P30 MH090333. 


\section{References}

1. Murphy SA. An experimental design for the development of adaptive treatment strategies. Statistical Methods 2005; 24:1455-81.

2. Lavori PW, Dawson R, J RA. Flexible treatment strategies in chronic disease: clinical research implications. Biological Psychology 2000; 48:605-614.

3. Stone RM, Berg DT, George SL, Dodge RK, Paciucci PA, Schulman P, Lee EJ, Moore JO, Powell BL, Schiffer CA. Granulocytemacrophage colony-stimulating factor after initial chemotherapy for elderly patients with primary acute myelogenous leukemia. The New England Journal of Medicine 1995; 332:1671-1677.

4. Stone RM, Berg DT, George SL, Dodge RK, Paciucci PA, Schulman P, Lee EJ, Moore JO, Powell BL, Baer MR, Bloomfield CD, Schiffer CA. Postremission therapy in older patients with de novo acute myeloid leukemia: a randomized trial comparing mitoxantrone and intermediate-dose cytarabine with standard-dose cytarabine. Blood 2001; 98:548-553.

5. Lunceford JK, Davidian M, Tsiatis AA. Estimation of survival distributions of treatment policies in two-stage randomization designs in clinical trials. Biometrics 2002; 58:48-57.

6. Robins JM, Rotnitzky A, Zhao LP. Estimation of regression coefficients when some regressors are not always observed. Journal of American Statistical Association 1994; 89:846-866.

7. Wahed AS, Tsiatis AA. Optimal estimator for the survival distribution and related quantities for treatment policies in two-stage randomization designs in clinical trials. Biometrics 2004; 60:124-133.

8. Wahed AS, Tsiatis AA. Semi-parametric efficient estimation of the survival distribution for treatment policies in two-stage randomization designs in clinical trials with censored data. Biometrika 2006; 93:147-161.

9. Guo X, Tsiatis AA. A weighted risk set estimator for survival distributions in two-stage randomization designs with censored survival data. The International Journal of Biostatistics 2005; 1:1-15.

10. Schmittlein DC, Morrison DG. The median residual lifetime: a characterization theorem and an application. Operations Research 1981; 29(2):392-399.

11. Haines AL, Singpurwalla ND. Some contributions to the stochastic characterization of wear. In Reliability and Biometry, Proschan F, Serfling RJ (eds). SIAM: Philadelphia, 1974.

12. Arnold $\mathrm{BC}$, Brokett PL. When does the $\beta$ th percentile residual life function determine the distribution? Operations Research 1983; 31:391-396.

13. Joe H, Proschan F. Percentile residual life functions. Operations Research 1984; 32:668-678.

14. Joe H. Characterizations of life distributions from percentile residual lifetimes. Annals of the Insititue for Statistical Mathematics $1985 ; 37: 165-172$.

15. Song JK, Cho GY. A note on percentile residual life. Sankhya 1995; 57:333-335.

16. Csorgo M, S C. Estimation of percentile residual life. Operations Research 1987; 35:598-605.

17. Chung CF. Confidence bands for percentile residual lifetime under random censorship mode. Journal of Multivariate Analysis 1989; 29:94-126.

18. Feng Z, Kulasekera KB. Nonparametric estimation of the percentile residual life function. Communications in StatisticsTheory and Methods 1991; 20:87-105.

19. Lillo RE. On the median residual lifetime and its aging properties: a characterization theorem and applications. Naval Research Logistics 2005; 4:370-380.

20. Holland PW. Statistics and causal inference. Journal of the American Statistical Association 1986; 81:945-60.

21. Cole SR, Frangakis CE. The consistency statement in causal inference: a definition or an assumption? Epidemiology 2009; 20(1):3-5.

22. Jeong J, Jung S, Costantino J. Nonparametric inference on median residual lifetimes in breast cancer patients. Biometrics $2008 ; \mathbf{6 4}: 157-163$

23. Stefanski LA, Boos DD. The calculus of m-estimation. The American Statistician 2002; 56(1):29-38.

24. Kidwell KM, Wahed AS. Weighted log-rank statistic to compare shared-path adaptive treatment strategies. Biostatistics $2013 ; \mathbf{1 4}(2)$

25. Ko JH. Statistical issues in the design and analysis of sequentially randomized trials. Ph.D. Thesis, Department of Biostatistics, University of Pittsburgh, 2010. 